Journal of Communications and Information Networks, Vol.2, No.4, Dec. 2017

DOI: $10.1007 / \mathrm{s} 41650-017-0040-8$

(C) Posts \& Telecom Press and Springer Singapore 2017

\title{
Energy saving for data centers using spatial multichannel optical wireless communication
}

\author{
Judy Kupferman*, Shlomi Arnon \\ Ben Gurion University of the Negev, Beer-Sheva 8410501, Israel \\ *Corresponding author, Email: judithku@post.bgu.ac.il
}

\begin{abstract}
Data centers are crucial elements in modern information technology. In order to implement the next generation of data centers, new challenges must be overcome. These include reducing the energy consumption, increasing the data rates, reducing the communication latency, increasing the flexibility and scalability, and reducing the maintenance time and cost. One promising way for meeting these challenges is to employ multichannel optical wireless communication as part of the data center hybrid communication network. In this paper, we analyze three technologies that could be used in this context: MIMO (Multiple-in-Multipleout), multiplexing through orbital angular momentum of light, and direct modulation through the large number of modes associated with orbital angular momentum. Our results indicate that these technologies could provide an innovative and flexible means of meeting the challenges of the next generation of data centers.
\end{abstract}

Keywords: data center, orbital angular momentum, optical wireless communication, energy consumption

\section{Introduction}

In recent years, society has been revolutionized by the increasing leaps in information and communication technologies. This began with the internet and then was followed by the smartphone. These tremendous advances gave rise to new horizons in social media, cloud computing, and bigdata processing. The applications range from accessories such as wireless watches to life-changing devices, with examples including the IoT (Internet of Things) and IoV (Internet of Vehicles), communication aids provided by Facebook, WhatsApp, Instagram, Snapchat, WeChat, and Imo, big-data analysis, 3D printing, multiplayer games, virtual reality, augmented cognition, and a plethora of other unforeseen applications. A DC (Data Center) is a cen- tralized warehouse, either physical or virtual, employing tens of thousands of servers that accumulate, store, process, and disseminate information and data. All this requires enormous computing power and infrastructure and consumes vast amounts of energy. The energy consumption of DCs today represents about $1.5 \%$ of the total humanity's electric energy consumption, with the predicted growth of that number up to $50 \%$ in $2020^{[1]}$. In addition to the energy-related challenge, the next generation of DCs will face the requirements to increase their data rates (the network throughput may already be as large as $\left.1 \mathrm{~PB} / \mathrm{s}^{[2]}\right)$, to reduce the communication latency, to increase the flexibility and scalability, and to reduce the maintenance time and cost (see Fig. 1).

A possible solution for this can be the use of a hybrid communication system including fiber 


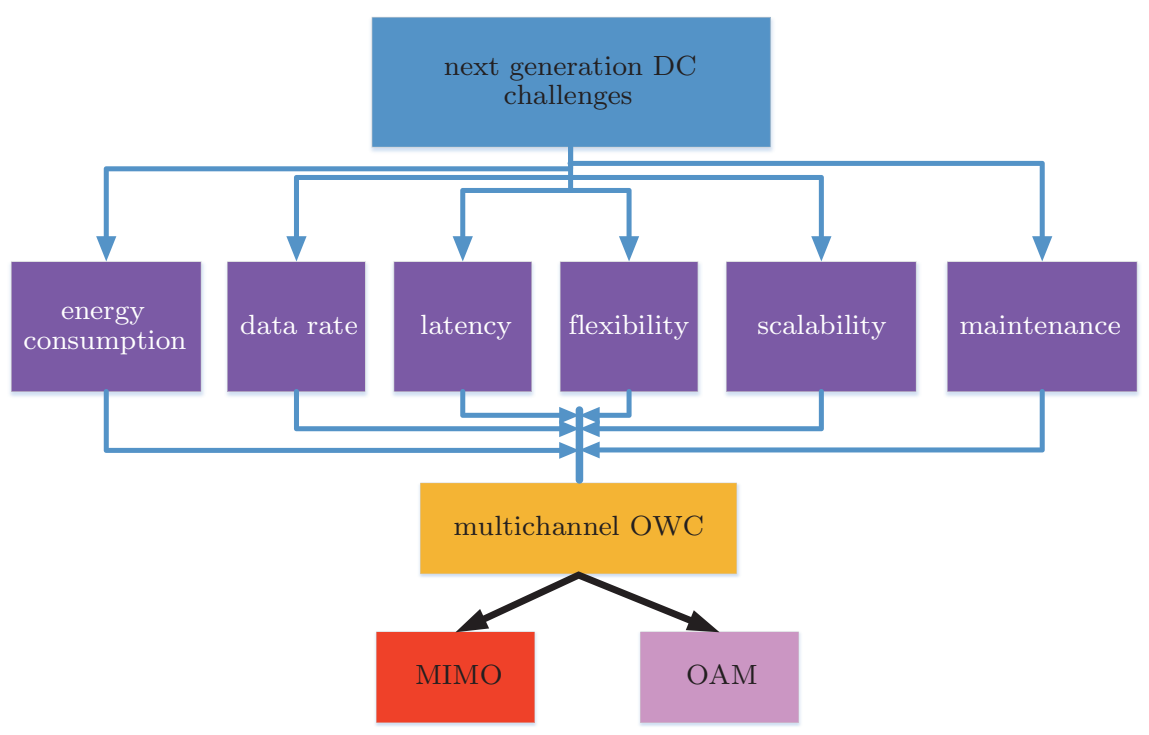

Figure 1 Challenges for next generation DCs and methods for meeting them

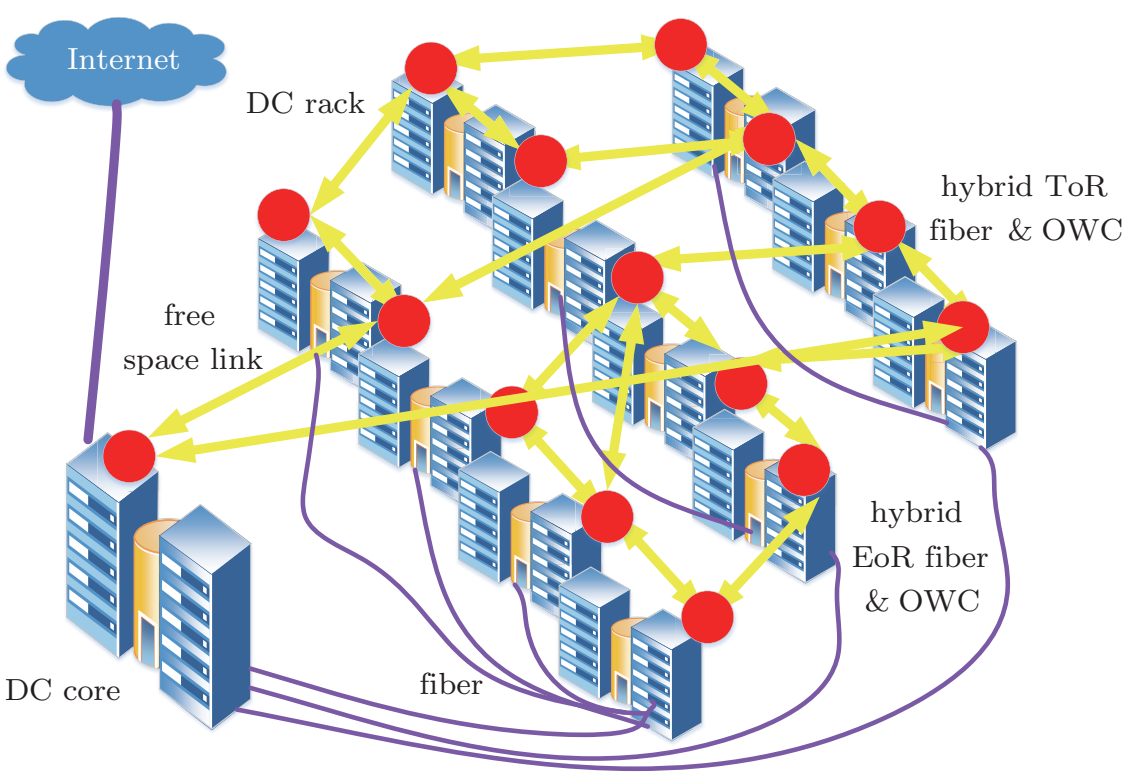

Figure 2 A proposed scheme for hybrid fiber/OWC communication in a DC

and OWC (Optical Wireless Communication) $)^{[3-7]}$ (Fig. 2). Such a system can provide great flexibility for various DC traffic patterns ${ }^{[8-10]}$, high bandwidth, high data rates, low latency ${ }^{[6]}$, and substantially reduced power consumption. OWC brings many advantages to this area. It can provide increased cooling efficiency (more than 30\% of the DC energy is consumed by air conditioning). It can minimize and simplify the building structure, as no raised floors or double-layered ceilings would be required for the net- work. An OWC system can have increased flexibility since it is not bound by the fiber infrastructure limits. The network topology can be changed quickly, rerouting workloads (live migration) from partially loaded servers and enabling idle servers to shut down, which would lead to significant energy reduction by reducing server operation and cooling. An optimal system could be hybrid, where fiber is used for longhaul links. This would be useful for communication between different groups of racks, as the new gener- 
ation of DCs will have tens of thousands of servers, covering huge areas of space. Within clusters of racks and between the racks the communication links could be OWC. These links could be rerouted depending on system needs and the identification of idle servers at any point in time, thus providing an agile and adaptable topology that could also lead to considerable savings in energy expenditure.

In addition, energy consumption could be further reduced through the use of spatial multichannel OWC. Such systems include MIMO systems, multiplexing using the OAM (Orbital Angular Momentum) of light, and direct modulation through OAM. Light with OAM can be produced in a large number of modes, thus offering faster data rate with a larger number of bits per symbol. It is clear that a spatial multichannel system could be combined with multiple other methods such as WDM and high-order OFDM to improve performance. Spatial multichannel OWC is ideally suited to DCs. Communication links are short-range, and the environment is controlled.

In this paper, we analyze the power consumption of spatial multichannel OWC. The rest of the paper is organized as follows. In the next section, the general layout of DCs is described, outlining the need for energy saving and increase in channel capacity. Then, the effect of multiple channels for increasing channel capacity is demonstrated. Following that, three options for achieving multichannel communication are analyzed, including MIMO, OAM multiplexing, and OAM direct detection.

\section{OWC in DCs}

A DC is a warehouse containing tens of thousands of servers, communication and storage equipment, and a support infrastructure that includes air conditioning and electricity regulator units. Normally, security equipment such as surveillance monitors is also necessary. Cabling may run under raised floors or in ceiling ducts. A typical DC can be comparable in size to a major football stadium. The servers are placed in racks. A rack might include as many as
96 servers $^{[11]}$. The racks are placed in rows. The physical design of a DC layout must consider energy conservation and minimization of heating. USA DC energy consumption was about 91 billion $\mathrm{kW} \cdot \mathrm{h}$ in 2013 and is projected to reach 140 billion $\mathrm{kW} \cdot \mathrm{h}$ in $2020^{[12]}$. Thus, energy saving and heat reduction are major concerns in the physical design of DCs. The reduction of energy consumption today is normally approached as follows. The aim is to prevent the heat produced by the servers from being recirculated and to separate hot and cold airstreams. A common layout uses a hot/cold aisle structure. Server racks are lined up in alternating rows, so that the cold air intakes face one way (cold aisles) and draw cold air into the racks to cool the devices (servers, switches, etc.), while the hot air exhausts face the other way (hot aisles). In order to isolate the hot and cold aisles from each other, a cold air containment system can be used to contain the air-conditioned air sent into the cold aisles and ensure that only that air flows into the air intakes of the devices. This could be a physical barrier separating the aisles, which could be walls, end-of-row doors, or sheets of vinyl plastic or Plexiglass. Cold air can be distributed through a raised floor, which can have perforated as well as solid tiles placed so that the amount of cooling is suitable to the servers in that location. The other technique is hot aisle containment, which ensures that the precision air conditioning units receive only hot air from the aisles ${ }^{[13]}$. Recommended widths of the hot aisles range from about one to two meters (three to six feet $)^{[14]}$.

OWC can prove a valuable method of communication in a DC. Intra-DC networks can have various topologies ${ }^{[15]}$, but essentially the information goes through a hierarchy of layers: the lowest layer is found in ToR (Top of Rack) switches on each server rack, EoR (End of Rack) switches at the end of each server row, and core switches at the highest layer. The next generation of DCs will require highspeed inter-rack communication links. A hybrid ToR would include fiber ports and an OWC transceiver and ports. It could communicate with the nearby and distant ToRs using an OWC link and to EoR 
by a fiber or an OWC link. This concept creates a dynamic and adaptive wireless network, which serves as an enhancement to the conventional fiber network. The OWC transceiver port includes a laser transmitter, optics, a detector, and electronics. The optical signal from the laser is collimated by the optics and emitted in the required direction. The received optical signal is filtered by an optical filter and concentrated by suitable optics to the detector. The signal is amplified by a TIA (Trans-Impedance Amplifier). The electronic system demodulates the information from the TIA output, and the digital data is fed into the ToR port. The OWC transceiver is directed to the appropriate direction based on the information from the network controller using gimbals (see Fig. 2).

There are various possibilities for the employment of optical wireless communication to improve DC communications, and here we analyze three of them. Each involves parallel communication and increased channel capacity. This leads to energy saving, as will be explained in the following section.

\section{Multichannel communication}

It is well known that the information-theoretic capacity for AWGN (Additive White Gaussian Noise) channel for large SNR is given by ${ }^{[16]}$

$$
C_{\mathrm{AWGN}}=W \mathrm{lb}\left(1+\frac{P}{N_{0} W}\right),
$$

where $W$ is the bandwidth, $N_{0}$ is the noise power density, and $P$ is the signal power for a single channel. This model is valid for signal-independent (additive) noise as in situations where the thermal noise or background are the dominant source of noise. However, the model can serve as a useful approximation even in the case of signal-dependent noise such as shot noise ${ }^{[17]}$.

The second term in the logarithm argument is much greater than 1, so the expression can be simplified to $C_{\mathrm{AWGN}}=W \mathrm{lb}\left(P /\left(N_{0} W\right)\right)$. Since the signal power appears in the logarithm argument, transmitting more information requires an exponential increase in signal power, and thus a much larger energy.
Use of multiple channels can increase the channel capacity without requiring a correspondingly large increase in energy. This can be explained in the following way. We want to find the power necessary to scale up the single link speed by a factor of $M$. If we increase the channel capacity by a factor of $M$, the total power $P_{S M}$ is exponentially larger than the original power $P$ :

$$
P_{S M}=N_{0} W\left(2^{C / W}\right)^{M} \approx P^{M} .
$$

The second way to scale up the link speed by a factor of $M$ is by increasing the capacity of parallel links. In that case the capacity for $M$ links is given by

$$
M C_{\mathrm{AWGN}}=M W \mathrm{lb}\left(\frac{P}{N_{0} W}\right),
$$

so that the total power for $M$ links, $P_{M M}$, is equal to $M$ times $P$ and is increased linearly by a factor of $M$. Fig. 3 shows the total power as a function of normalized channel capacity. The purple bars show the increase in total power with increasing capacity for a single channel link. The yellow bars show the increase in total power with increasing capacity.

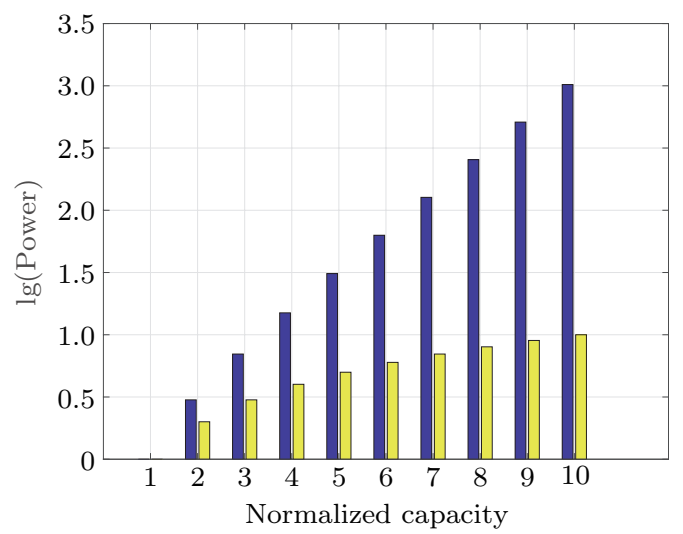

Figure 3 Total power as a function of normalized channel capacity

Optical communication is advantageous over $\mathrm{RF}$ communication for the following reason. The maximum number of spatial channels with efficient coupling in an FSO link is approximately ${ }^{[18]}$

$$
M_{\max } \approx \frac{A_{T} A_{R}}{\lambda^{2} z^{2}},
$$




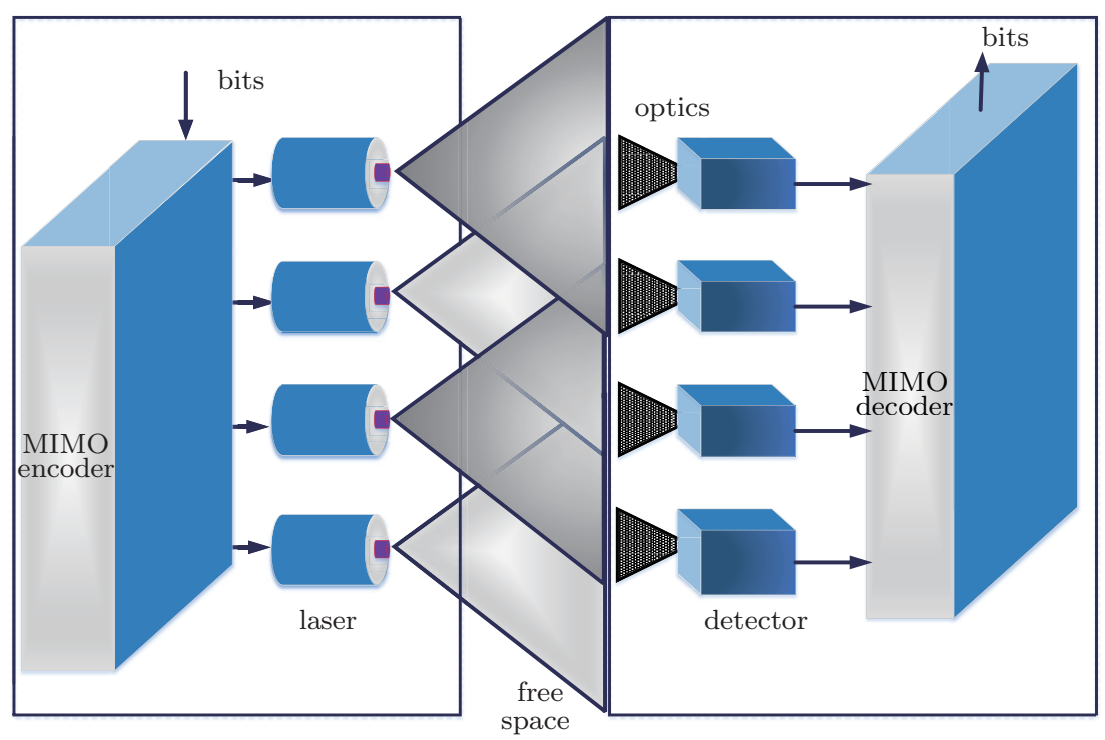

Figure 4 A schematic for a MIMO system

where $A_{T}$ and $A_{R}$ are the transmitter and receiver areas, respectively, $z$ is the separation distance between the transmitter and receiver, and $\lambda$ is the optical wavelength. For example, for transmitter and receiver areas of $0.1 \mathrm{~m}$, separation distance of $10 \mathrm{~m}$, optical wavelength of $\lambda=1550 \mathrm{~nm}$, and RF frequency of $60 \mathrm{GHz}^{[19]}$, the number of channels will be $M_{\text {max_OwC }} \approx 4 \times 10^{7}$, while $M_{\text {max_RF }} \approx 4$. Thus, we see that an optical wireless system could support six orders of magnitude more spatial channels than an RF system.

\section{Practical implementations}

We now analyze three technologies for implementation of multichannel communication.

\subsection{MIMO}

The first technology is the MIMO system. Such a system includes an array of transmitters and a similar array of receivers. These enable simultaneous multipath communication between the elements of the receiver and transmitter arrays ${ }^{[20,21]}$. The signal is multi-dimensionally analyzed and decoded at the receiver end. The channel capacity is increased in proportion to the number of emitters. MIMO can be used in both wireless and fiber systems, and it can be used in conjunction with spatial multiplexing, where a high-rate signal is split into multiple streams of lower-rate signals sent in parallel. Fig. 4 shows a schematic for such a system. The signal bits are fed into a modulator and sent by separate transmitters through space to the receiver, shown on the right. At the receiver end, there is an array of detectors corresponding to the separate transmitters. These could be, for example, p.i.n. photodetectors in TIA configurations. At the receiver, the light beams reach an optical system, which sends them to the detectors and then to a MIMO decoder. The gray triangles in the figure indicate overlap in the transmitted beams. Note that the transmitter laser array is shown in the figure as cylinders, while the detector receiver array is shown as cubes. This is because there will be some overlap of the transmitted beams at the detector array, and signal processing is necessary in order to isolate and distinguish different beams.

\subsection{OAM theory}

Another possibility for increasing the channel capacity is through the use of the OAM of light. This can be implemented in a number of ways, as detailed below, and can also be integrated into MIMO systems. But the exciting innovation in the use of OAM is the exploitation of a previously unexplored characteris- 
tic of light.

In our work, we define OAM using a complete basis for free-space electromagnetic waves comprised of LG (Laguerre-Gaussian) mode sets. These have two parameters, radial $(p)$ and azimuthal $(l)$. The azimuthal parameter gives the orbital angular momentum $l \hbar$ per photon (where $l$ is the topological charge and $\hbar$ is Planck's constant $h$ divided by $2 \pi$ ). Some other works treat OAM as a set of modes with $p=0$, which is a subset of the LG family of modes.

It is well known that light can carry angular momentum ${ }^{[22,23]}$. Following the work of Allen et al. ${ }^{[24]}$, it was quickly realized that OAM modes of light could be an effective way to increase bandwidth in optical communication, and corresponding methods of detection and multiplexing were soon invented and explored. A comprehensive survey of the application of OAM to communications is found in Ref. [25], with details of beam generation, multiplexing, transmission through free space and fiber, networking, and use in quantum information.

The solution to the wave equation in vacuum in the paraxial approximation in cylindrical coordinates is given by an infinite set of LG modes. While other types of light beams having OAM can be produced, $K_{\text {Kahn }}{ }^{[21]}$ showed that LG beams are the most efficient for this purpose. A paraxial beam of light can be decomposed into LG modes. Allen et al. showed that these modes have orbital angular momentum. They have amplitude $A_{k l p}(\vec{r})$ and phase $\Theta_{k l p}(\vec{r})$ as follows ${ }^{[26]}$ :

$$
\begin{aligned}
A_{k l p}(\vec{r})= & \frac{N_{l p}}{w_{0} \sqrt{1+z^{2} / z_{R}^{2}}}\left(\frac{\sqrt{2} r}{w(z)}\right)^{|l|} L_{p}^{|l|}\left(\frac{2 r^{2}}{w(z)^{2}}\right) \\
& \cdot \mathrm{e}^{-r^{2} / w(z)^{2}}, \\
\Theta_{k l p}(\vec{r})= & \frac{k r^{2} z}{2\left(z^{2}+z_{R}^{2}\right)}+l \phi \\
& +(2 p+|l|+1) \operatorname{arctg}\left(\frac{z}{z_{R}}\right)+k z
\end{aligned}
$$

where $w(z)$ is the beam width at the axial coordinate $z, z_{R}$ is the Rayleigh length with $w^{2}(z)=$ $2\left(z^{2}+z_{R}^{2}\right) / k z_{R}, N_{l p}=\sqrt{2 p ! / \pi(|l|+p) !}$ is a normalization constant, $L_{p}^{|l|}$ is the associated Laguerre polynomial, and $A_{k 00}$ is the amplitude of a plane wave propagating along the $z$ axis with wave vector $k$. Fig. 5 shows the beam patterns of six different Laguerre-Gauss modes.

The azimuthal and radial indices, $l$ and $p$, appear in both amplitude and phase. For communication purposes, existing works on OAM most often make use of the azimuthal parameter $l$. For long-haul links, this is done because the modes with $p=0$ have the lowest beam divergence. In Ref. [27] an expression is derived for the spot size, that is, the beam size large enough to contain all of the intensity maxima:

$$
\begin{gathered}
\sigma_{r}(z)_{p l}=w(z) \sqrt{1+l+2 p}, \\
w(z)=w_{0}\left[1+\left(\frac{\lambda z}{\pi w_{0}^{2}}\right)^{2}\right]^{1 / 2},
\end{gathered}
$$

where $z$ is the distance from the source, $w_{0}=\lambda z_{R} / \pi$ and $z_{R}$ is the Rayleigh length. The radial parameter has a stronger effect on the spot size. However, the inter-rack communication links in DCs are shortrange, as the distance between the racks is usually a meter or two. Therefore, the beam divergence is not a major consideration. Fig. 6 shows the radial intensity profile for several beams. The peaks in the figure indicate the rings of light. With increasing radial index, the number of rings grows and the beam radius becomes larger. In the figure, $z=10 \mathrm{~m}$ and $\lambda=1550 \mathrm{~nm}$. As the radial parameter increases, so do the number of concentric rings of light, which appear as peaks of the graph, and the beam radius grows accordingly. With $p$ as large as 5, at a distance of $10 \mathrm{~m}$, the beam radius without any converging optics is $2 \mathrm{~cm}$.

Another disadvantage in long-haul links can be the difficult alignment of the transmitters and receivers. One ingenious solution involves transformation of OAM encoded states into hybrid states of OAM and polarization that are rotationally invariant and inverse transformation at the receiving end back to the OAM states, which are then decoded ${ }^{[28]}$. Another possible problem is turbulence ${ }^{[29]}$. None of these problems are serious in DC inter-rack links, because the involved distances are short, and the environment is accurately controlled and cooled. OAM 

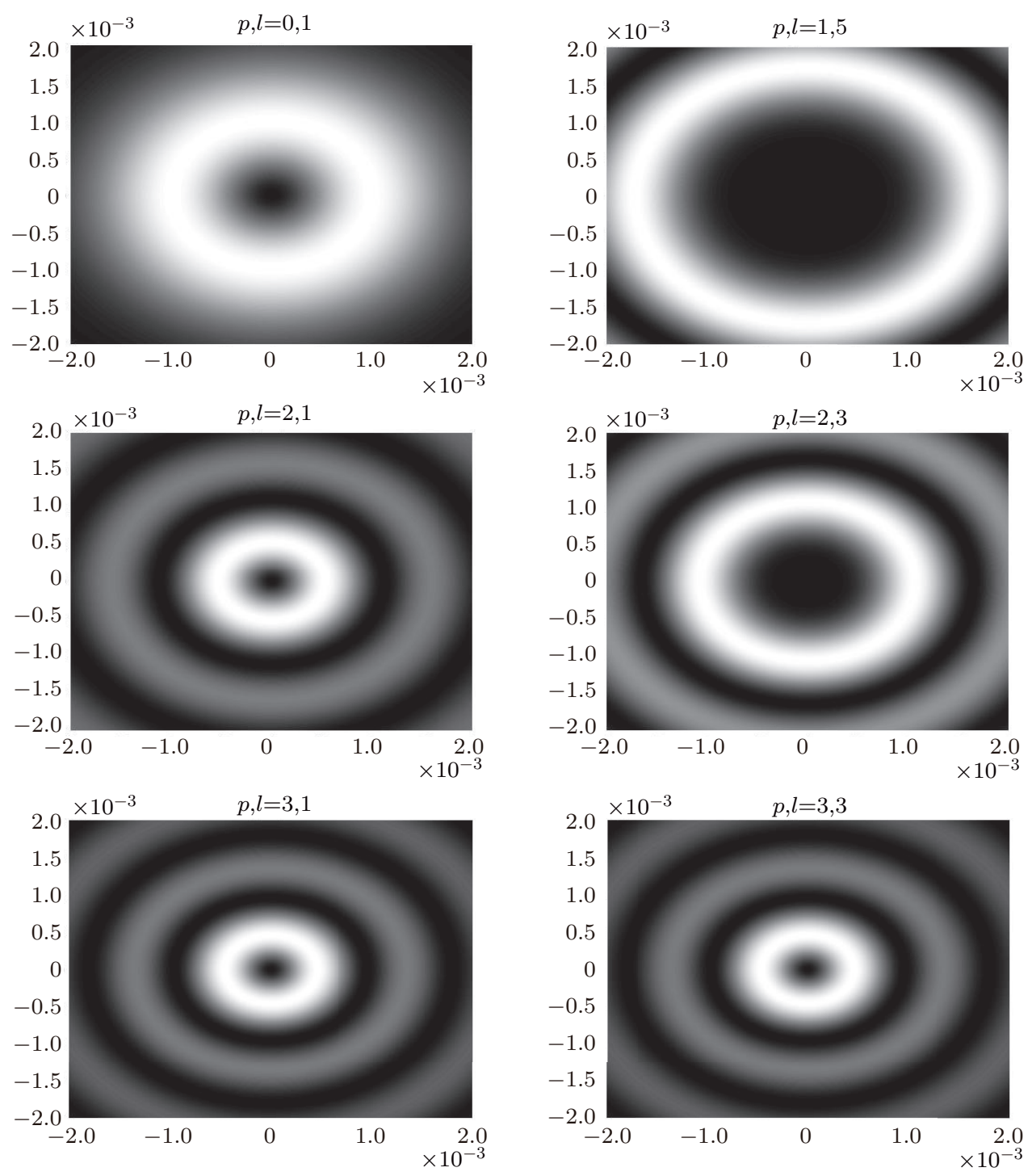

Figure 5 Beam patterns for six different Laguerre-Gauss modes

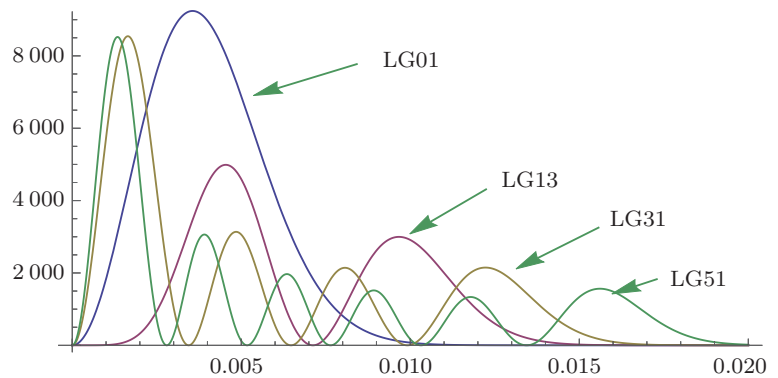

Figure 6 Radial intensity distribution for four different LG modes: $(p, l)=(0,1) ;(p, l)=(1,3) ;(p, l)=(3,1)$, and $(p, l)=(5,1)$

modes can be generated in a variety of ways including holograms, cylindrical lens converters, phase plates, SLMs (Spatial Light Modulators), and DMDs (Digital Mirror Devices) ${ }^{[25]}$. At the receiving end, the beams can be analyzed in three different ways: by reverse encoding, either with holograms or with SLMs, by reversing the received signal, or by direct detection and image processing. In addition, a specialized sorter has been developed ${ }^{[30]}$, which makes use of optical geometric transformations to efficiently separate each mode into a specified location on the detector.

There are two basic ways to exploit OAM for increasing channel capacity. The first is OAM multiplexing. 

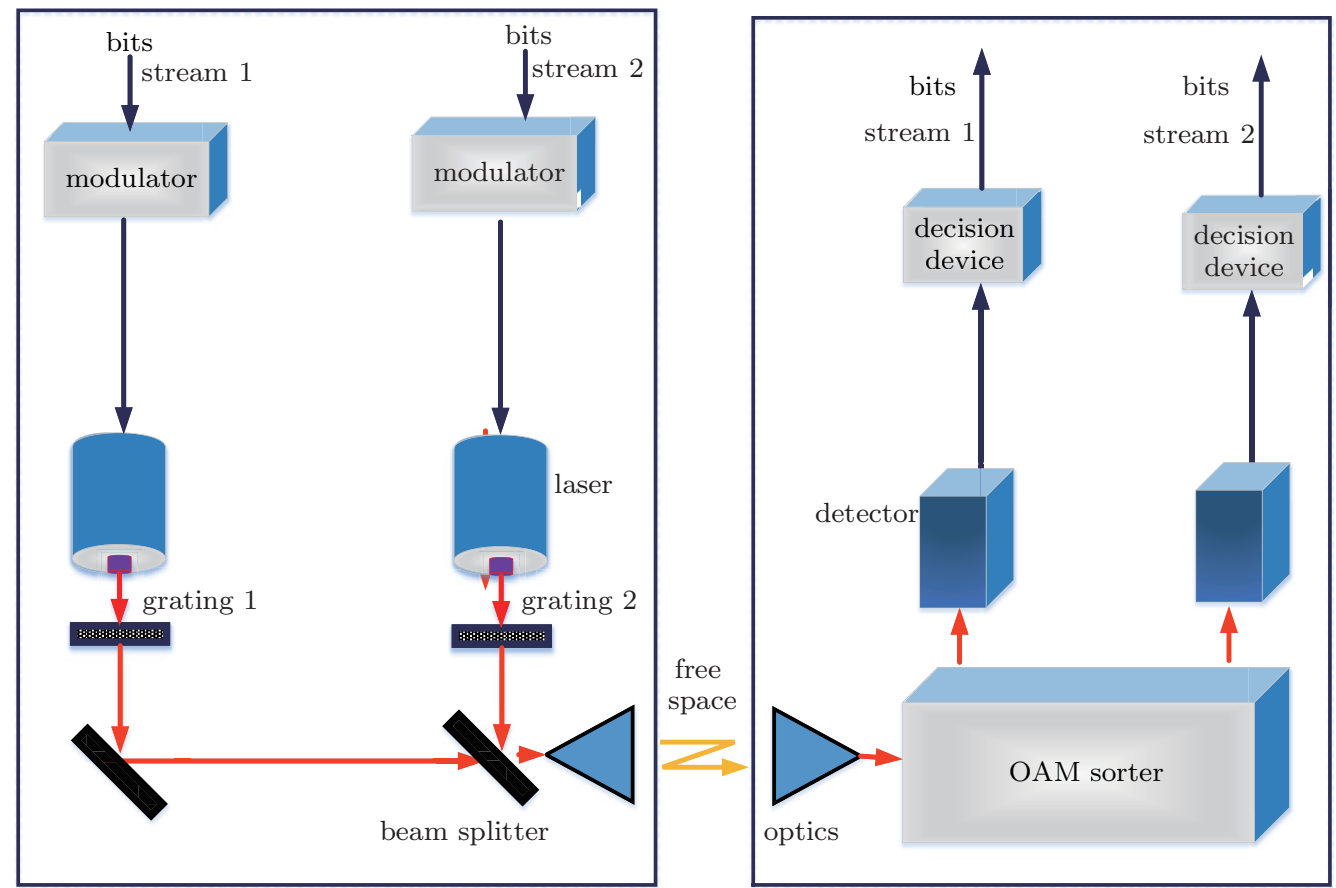

Figure 7 OAM multiplexing scheme

\subsection{OAM multiplexing}

An OAM multiplexing scheme is shown in Fig. 7. Two streams of bits are fed into a modulator. Each bit stream modulates a laser beam. The laser beam then goes through a grating that transforms the light into one of a set of orthogonal OAM modes (the grating could be, for example, an SLM or a phase plate). These orthogonal OAM beams are then multiplexed so that one combined beam is emitted to free space. At the receiver, the modes are sent into an OAM sorter and are demultiplexed to transmit the desired information.

OAM multiplexing is problematic in fiber systems due to mode coupling, although special fibers have been developed for this purpose ${ }^{[31]}$. However, OAM multiplexing is clearly suitable for the type of shortrange OWC outlined in this paper. Since the copropagating OAM modes are orthogonal, they can be transmitted and then efficiently separated at the receiving end. This can be done using a number of techniques, some of which have been demonstrated in the laboratory (beam splitters, holograms), but are too cumbersome for our purposes. More promis- ing options that are being explored include mode sorters, photonic integrated circuits, and direct detection with image processing ${ }^{[25]}$. OAM multiplexing can be combined with other forms of multiplexing such as PDM and WDM to further increase capacity, as was shown in Ref. [32], with the capacity reaching $100 \mathrm{Tbit} / \mathrm{s}$. Recently, multiplexing of LG modes using both radial and azimuthal degrees of freedom was done for over 100 modes in a proof-of-principle experiment ${ }^{[33]}$. Some studies took advantage of NOMA (Non-Orthogonal Multiplexing Access) $)^{[34,35]}$ and schemes combining both integer and fractional OAM charges ${ }^{[36]}$.

\subsection{OAM modulation}

Another possibility for using OAM takes advantage of the large amount of LG modes to provide a much larger alphabet for encoding. Here, each mode represents a different data symbol. Fig. 8 shows a typical scheme. At the transmitter, a bit stream is input and the modulating device sends signals to four lasers. The light from each laser goes through a grating that transforms it to an OAM mode correspond- 

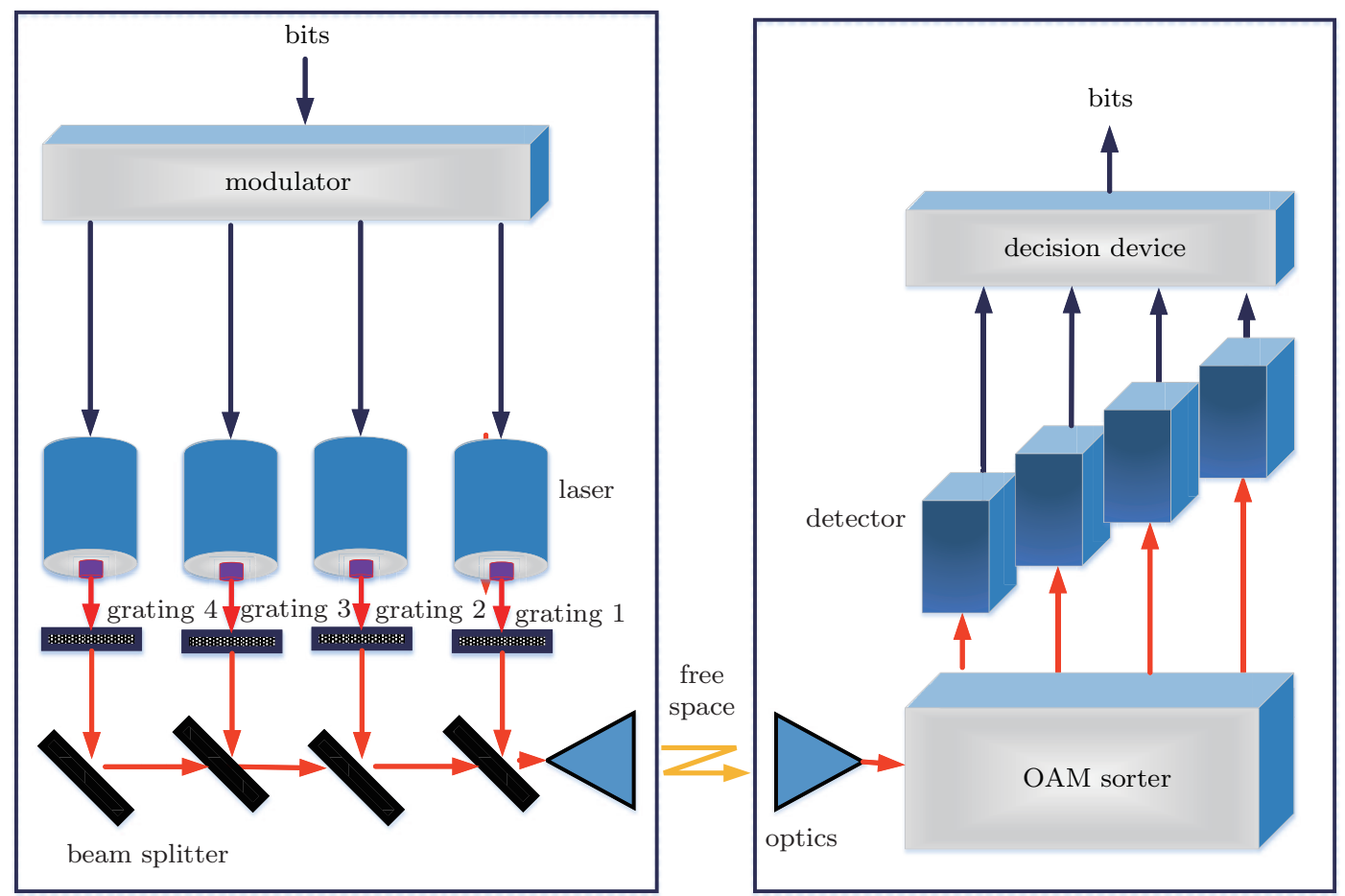

Figure 8 OAM modulation scheme

ing to the desired symbol. The light is then directed to a telescope and sent to the receiver, where it is sent through an optical system to a sorter, and the respective modes are detected and identified. A decision unit then outputs the relevant symbols, and the information is recovered.

In this way, $n$ different modes represent $n$ different data symbols. The number of modes that can be achieved practically is extremely large. Experiments have generated over 300 OAM modes, having $l>300$ even with the radial index $p=0$ to contain beam divergence ${ }^{[37,38]}$. Thus, LG modes can in theory provide an extremely long alphabet for signal transmission, even if one prefers to use only the azimuthal parameter, with $p=0$ for smaller divergence angles. The signals can then be analyzed at the receiving end using image processing. This could be done using a detector matrix, which is then compared to a prepared numerical dictionary of matrices to extract the correct signal. A more sophisticated implementation could use a specialized detector that takes advantage of the circular symmetry of the OAM beam ${ }^{[39]}$.

\section{Power requirements for OAM vs. MIMO}

In this section, we compare the power consumption of the two proposed schemes: OAM and MIMO. According to Ref. [21] the approximate number of independently addressable sub-channels, that is, the approximate number of sub-channels to be addressed without crosstalk has an upper limit of

$$
M_{\text {MIMO_max }} \leqslant 0.9 L^{2},
$$

where $L$, the physical transmission factor, is a dimensionless parameter defined as a function of the transmitter/receiver aperture, the numerical aperture, and the wavelength.

The approximate number of independently addressable OAM sub-channels (defined as SMM in Ref. [21]) is upper-bounded as

$$
M_{\text {OAM_max }} \approx \frac{1}{\sqrt{2}}(L+7)(L-1), L>9 .
$$

From Eqs. (1) and (9), and for a given requirement of capacity $C_{R}$, the required transmitter power for a 
MIMO system is given by

$$
P_{\mathrm{MIMO}} \approx\left(2^{C_{R} /\left(W 0.9 L^{2}\right)}-1\right) N_{0} W .
$$

From Eqs. (1) and (10), and for a given requirement of capacity $C_{R}$, the required transmitter power for an OAM system is given by

$$
\left.P_{\mathrm{OAM}} \approx\left(2^{C_{R} /\left(W \frac{1}{\sqrt{2}}(L+7)(L-1)\right.}\right)-1\right) N_{0} W, L>9 .
$$

The logarithmic ratio between $P_{\mathrm{OAM}}$ and $P_{\mathrm{MIMO}}$ from Eqs. (11) and (12) is given approximately by

$$
\begin{aligned}
L R_{\text {Power }} & =\mathrm{lb}\left(\frac{P_{0 A M}}{P_{\text {MIMO }}}\right) \\
& \approx \frac{C_{R}}{W}\left(\frac{\sqrt{2}}{(L+7)(L-1)}-\frac{1}{0.9 L^{2}}\right), L>9 .
\end{aligned}
$$

In Fig. 9, we calculate numerically the exact logarithm power ratio for $L>9$ with $N_{0}=1$ for different values of $C_{R} / W$. For all three curves, it is easy to see that, for the low values of $L$, the use of an OAM system reduces the power consumption dramatically in comparison to a MIMO system. The effect is most pronounced for the highest value of $C_{R} / W$. At higher values of $L>20$, MIMO systems show a small and robust advantage over OAM systems, while the effect of $C_{R} / W$ is negligible.

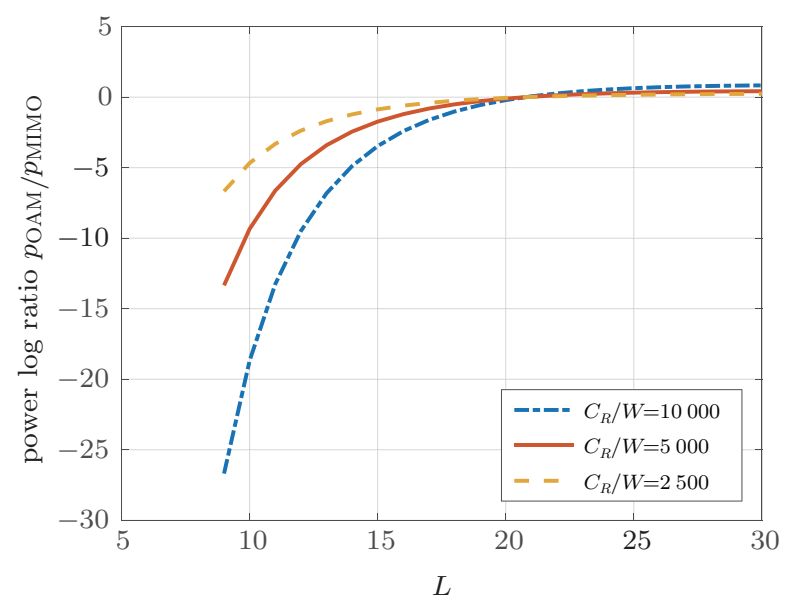

Figure 9 The logarithmic power ratio as a function of $L$ for different ratios of the channel capacity to the bandwidth

\section{Summary and conclusions}

Increase in channel capacity could make a significant contribution to reduction of energy cost in DCs. We have presented several approaches for accomplishing this: using parallel propagation with MIMO, multiplexing of orbital angular momentum of light, and using the large mode alphabet of OAM for encoding signals. Comparison of MIMO and OAM in terms of power requirements depends on the system being designed. For low physical transmission factors, OAM shows a clear advantage in reducing the power consumption. For high physical transmission factors, MIMO is slightly preferable.

Here, we compare MIMO and OAM only in terms of transmitter power. A comprehensive study would need to compare static and dynamic energy consumption for the digital and analog electronic subsystems of the communication system. However, in many cases, the transmitter power represents a dominant contribution to the total energy consumption ${ }^{[40-42]}$. Therefore, our analysis provides a qualitative description of the advantages of different schemes.

Naturally, these methods can also be combined for obtaining an even more efficient system. In addition, a combination of OAM and polarization can be employed as an even more flexible method of encoding signals. Integration of these methods in a hybrid fiber/OWC communications scheme is ideally suited for the controlled environment and addressing the growing challenges of the next generation of DCs.

\section{References}

[1] D. Boru, D. Kliazovich, F. Granelli, et al. Energyefficient data replication in cloud computing datacenters [J]. Cluster computing, 2015, 18(1): 385-402.

[2] A. Singh, J. Ong, A. Agarwal, et al. Jupiter rising: a decade of clos topologies and centralized control in google's datacenter network [J]. ACM SIGCOMM computer communication review, 2015, 45(4): 183-197.

[3] A. S. Hamza, J. S. Deogun, D. R. Alexander. Wireless communication in data centers: a survey $[\mathrm{J}]$. IEEE communications surveys \& tutorials, 2016, 18(3): 15721595. 
[4] S. Arnon, M. Uysal, Z. Ghassemlooy, et al. Optical wireless communications [J]. IEEE journal on selected areas in communications, 2015, 33(9): 1733-1737.

[5] Z. Ghassemlooy, S. Arnon, M. Uysal, et al. Emerging optical wireless communications-advances and challenges [J]. IEEE journal on selected areas in communications, 2015, 33(9): 1738-1749.

[6] S. Arnon. Next-generation optical wireless communications for data centers [C]//SPIE OPTO. International Society for Optics and Photonics, 2015: 938703.

[7] J. Kupferman, S. Arnon. Receiver design for OWC orbital angular momentum communication in data center applications [C]//10th IEEE International Symposium on Communication Systems, Networks and Digital Signal Processing (CSNDSP), 2016: 1-6.

[8] D. Halperin, S. Kandula, J. Padhye, et al. Augmenting data center networks with multi-gigabit wireless links [J]. ACM SIGCOMM computer communication review, 2011, 41(4): 38-49.

[9] I. Fujiwara, M. Koibuchi, T. Ozaki, et al. Augmenting low-latency hpc network with free-space optical links [C]//IEEE 21st International Symposium on High Performance Computer Architecture (HPCA), 2015: 390401.

[10] S. Arnon, M. Fridman. Data center switch based on temporal cloaking $[\mathrm{J}]$. Journal of lightwave technology, 2012, 30(21): 3427-3433.

[11] M. Vasudevan, Y. C. Tian, M. L. Tang, et al. Profilebased application assignment for greener and more energy-efficient data centers [J]. Future generation computer systems, 2016, 67: 94-108.

[12] K. Normandieu. Approaches to data center containment [EB/OL]. http://www.datacenterknowledge.com/ archives/2012/11/08/approaches-to-data-centercontainment/.

[13] J. Niemann. Best practices for designing data centers with the infrastruxure inrow RC [Z]. Application note of American Power Conversion, 2006.

[14] W. F. Xia, P. Zhao, Y. G. Wen, et al. A survey on data center networking(DCN): infrastructure and operations $[\mathrm{J}]$. IEEE communications surveys \& tutorials, 2017, 19(1): 640-656.

[15] J. G. Proakis. Digital communications [M]. New York: McGraw-Hill, 1995.

[16] J. M. Kahn, D. A. Miller. Communications expands its space [J]. Nature photonics, 2017, 11(1): 5-8.

[17] R. M. Gagliardi, S. Karp. Optical communications [M]. 2nd edition. Wiley Interscience, 1995.

[18] M. Z. Zaaimia, R. Touham, L. Talbi, et al. 60-GHz statistical channel characterization for wireless data centers $[\mathrm{J}]$. IEEE antennas and wireless propagation letters, 2016, 15: 976-979.

[19] H. Lipfert. MIMO OFDM: space-time coding spatial multiplexing, increasing performance and spectral efficiency in wireless systems, part I technical basis (tech- nical report) [R]. Institut fr Rundfunktechnik (2007).

[20] S. Arnon, J. Barry, G. Karagiannidis, et al. Advanced optical wireless communication systems [M]. Cambridge: Cambridge University Press, 2012.

[21] N. B. Zhao, X. Y. Li, G. F. Li, et al. Capacity limits of spatially multiplexed free-space communication $[\mathrm{J}]$. Nature photonics, 2016, 9(12): 822-826.

[22] J. D. Jackson. Classical electrodynamics [M]. 2nd edition. New York: John Wiley and Sons, 1975.

[23] F. Rohrlich. Classical charged particles [M]. World Scientific Publishing Co. Inc., 2007.

[24] L. Allen, M. W. Beijersbergen, R. J. Spreeuw, et al. Orbital angular momentum of light and the transformation of Laguerre-Gaussian laser modes [J]. Physical review A, 1992, 45(11): 8185-8189.

[25] A. E. Willner, A. F. Molisch, C. Bao, et al. Optical communications using orbital angular momentum beams [J]. Advances in optics and photonics, 2015, 7(1): 66-106.

[26] D. L. Andrews, M. Babiker. The angular momentum of light $[\mathrm{M}]$. Cambridge: Cambridge University Press, 2013.

[27] R. L. Phillips, L. C. Andrews. Spot size and divergence for Laguerre Gaussian beams of any order [J]. Applied optics, 1983, 22(5): 643-644.

[28] G. Vallone, V. D'. Ambrosio, A. Sponselli, et al. Freespace quantum key distribution by rotation-invariant twisted photons [J]. Physical review letters, 2014, 113(6): 060503.

[29] Y. X. Ren, H. Huang, G. D. Xie, et al. Atmospheric turbulence effects on the performance of a free space optical link employing orbital angular momentum multiplexing [J]. Optics letters, 2013, 38(20): 4062-4065.

[30] G. C. Berkhout, M. P. Lavery, J. Courtial, et al. Efficient sorting of orbital angular momentum states of light [J]. Physical review letters, 2010, 105(15): 153601.

[31] N. Bozinovic, Y. Yue, Y. X. Ren, et al. Terabit-scale orbital angular momentum mode division multiplexing in fibers [J]. Science, 2013, 340(6140): 1545-1548.

[32] H. Huang, G. D. Xie, Y. Yan, et al. 100 Tbit/s freespace data linkenabled by three-dimensional multiplexing of orbital angular momentum, polarization, and wavelength [J]. Optics letters, 2014, 39(2): 197-200.

[33] A. Trichili, C. Rosales-Guzmán, A. Dudley, et al. Optical communication beyond orbital angular momentum [J]. Scientific reports, 2016, 6: 27674.

[34] Y. Saito, Y. Kishiyama, A. Benjebbour, et al. Nonorthogonal multiple access (NOMA) for cellular future radio access $[\mathrm{C}] / / 2013$ IEEE 77 th Vehicular Technology Conference (VTC Spring), 2013: 1-5.

[35] Z. G. Ding, Z. Yang, P. Z. Fan, et al. On the performance of non-orthogonal multiple access in $5 \mathrm{G}$ systems with randomly deployed users [J]. IEEE signal processing letters, 2014, 21(12): 1501-1505.

[36] Y. Q. Zhao, X. Zhong, G. H. Ren, et al. Capacity of arbitrary-order orbital angular momentum multiplexing 
system [J]. Optics communications. 2017, 387: 432-439.

[37] R. Fickler, R. Lapkiewicz, W. N. Plick, et al. Quantum entanglement of highangular momenta $[\mathrm{J}]$. Science, 2012, 338(6107): 640-643.

[38] L. X. Chen, W. H. Zhang, Q. H. Lu, et al. Making and identifying optical superpositions of high orbital angular momenta [J]. Physical review A, 2013, 88(5): 053831.

[39] J. Kupferman, S. Arnon. Decision algorithm for orbital angular momentum receiver [Z]. arXiv:1703.07984, 2017.

[40] S. Saeedi, A. Emami. A $25 \mathrm{~Gb} / \mathrm{s} 170 \mu \mathrm{W} / \mathrm{Gb} / \mathrm{s}$ optical receiver in $28 \mathrm{~nm}$ CMOS for chip-to-chip optical communication $[\mathrm{C}] / /$ IEEE Radio Frequency Integrated Circuits Symposium, 2014: 283-286.

[41] F. Y. Liu, D. Patil, J. Lexau, et al. 10-Gbps, 5.3$\mathrm{mW}$ optical transmitter and receiver circuits in 40-nm CMOS [J]. IEEE journal of solid-state circuits, 2012, 47(9): 2049-2067.

[42] C. Li, R. Bai, A. Shafik, et al. Silicon photonic transceiver circuits with microring resonator bias-based wavelength stabilization in 65nm CMOS [J]. IEEE journal of solid-state circuits, 2014, 49(6): 1419-1436.

\section{About the authors}

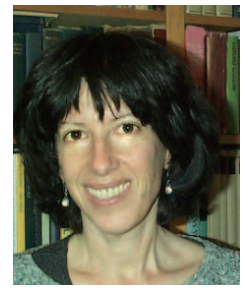

Judy Kupferman is currently a postdoc in the Electrical Engineering Department of Ben Gurion University. She is also a recognized expert in lighting design, which she taught for many years at Tel Aviv University. (Email: judithku@post.bgu.ac.il)

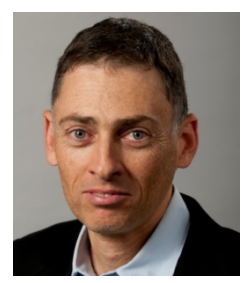

Shlomi Arnon is a professor at the Department of Electrical and Computer Engineering at Ben-Gurion University (BGU), Israel. Professor Arnon's honors and awards include SPIE Fellow and Fulbright Fellow. His research has produced more than eighty journal papers in the area of optical, satellite and wireless communication. (Email: shlomi@ee.bgu.ac.il) 